induced by these stimuli are idiopathic generalized epilepsies, especially juvenile myoclonic epilepsy, and the cortical mechanisms are complex, ultimately involving cortico-reticular or cortico-cortical pathways. (Ferlazzo E, Zifkin BG, Andermann E, Andermann F. Cortical triggers in generalized reflex seizures and epilepsies. Brain April 2005;128:700-710). (Respond: Benjamin G Zifkin MDCM FRCPC, Epilepsy Clinic, Montreal Neurological Institute and Hospital, 3801 University Street, Room 128, Montreal, QC H3A 2BA, Canada).

COMMENT. The authors hypothesize that patients with cortical triggers of GRS present regions of cortical hyperexcitability overlapping with the areas activated during sensory stimulation and cognitive or planned motor (praxis) activities. When these hyperexcitable areas are activated sufficiently, epileptic activity is produced that involves cortico-reticular or cortico-cortical pathways, resulting in a generalized reflex seizure. Genetic or acquired lesions may be responsible for the neuronal hyperexcitability.

Patiern sensitive epiiepsy, discussed in Ped Neur Briefs February 2005, has a higher incidence of focal symptomatic seizures than the generalized idiopathic reflex epilepsies. The EEG in pattern-sensitive epilepsies shows focal epileptiform discharges, whereas photosensitive epilepsies are usually accompanied by generalized polyspike-wave and spikewave complexes.

\title{
PAROXYSMAL TONIC UPGAZE
}

This oculomotor syndrome of childhood, originally termed "benign paroxysmal tonic upgaze of childhood" (Ouvrier and Billson, 1988) is reviewed from the Children's Hospital of Westmead, Sydney, NSW, Australia. Since the original description of 4 cases, a total of 49 cases have been reported. The clinical features listed in the original cases were as follows: onset before 1 year of age; conjugate upward deviation of the eyes, with neck flexion; downbeating compensatory saccades; normal horizontal eye movements; fluctuation of symptoms during the daytime and relief in sleep; exacerbation during febrile illness; intermittent or persistent ataxia; otherwise normal neurologic exam; no deterioration and eventual improvement with long-term follow-up; normal EEG, CT, and CSF neurotransmitters. Some subsequent reports have described etiological factors, including: genetic, autosomal dominant or recessive inheritance in 4 families; fetal valproate exposure in 3 cases; and cerebral abnormalities in 5 (hypomyelination ( 2 cases), periventricular leukomalacia, Vein of Galen malformation, pinealoma). About $40 \%$ have learning or mild cognitive deficits, and $10 \%$ are moderately to severely retarded. Several have a history of febrile convulsions, 2 had epilepsy, but the EEG shows no epileptic activity during the tonic upgaze. Apart from the few cases with structural pathology involving the upper brainstem, a localization-related lesion is not evident. About $50 \%$ of cases have a favorable outcome, $25 \%$ have residual ataxia, and $25 \%$ may have strabismus or nystagmus as a sequel. Treatment with

L-dopa is sometimes successful, but AEDs, including acetazolamide and ACTH, are of no benefit. (Ouvrier R, Billson F. Paroxysmal tonic upgaze of childhood - a review. Brain Dev April 2005;27:185-188). (Respond: Dr Robert Ouvrier, E-mail: roberto@chw.edu.au).

COMMENT. A tonic upgaze may be caused by a space-occupying lesion involving the brainstem, and an MRI is indicated in these infants, especially when ataxic. 